\title{
Generating chimeric spinal cord: a novel model for transplantable oligodendrocyte progenitors derived from embryonic stem cells
}

\author{
Sudhakar Vadivelu, B.S., B.A., Daniel Becker, M.D., \\ ANd John W. McDonald, M.D., Ph.D.
}

The International Center for Spinal Cord Injury, Kennedy Krieger Institute, and Department of Neurology, Johns Hopkins University School of Medicine, Baltimore, Maryland; and Department of Neurology, Vanderbilt University Medical Center, Nashville, Tennessee

\begin{abstract}
Object. To identify and evaluate stem cell-derived oligodendrocytes obtained for cell transplantation therapies, the authors developed a novel model to examine single, adult oligodendrocytes in situ.

Methods. Green fluorescent protein-expressing, mouse embryonic stem cells (ESCs) were neural induced and additionally staged in an oligosphere preparatory step for high-yield derivation of oligodendrocyte progenitors. These transplantable, induced progenitors were injected into postnatal Day 2 rat pups, in which spinal cord sections were then examined at 3 and 9 weeks posttransplantation.

Conclusions. Transplanted oligosphere ESCs survived and integrated anatomically into postnatal and adult white matter, generating targeted regions of chimeric spinal cord. A simple model for identifying adult oligodendrocytes in situ is presented, which is suitable for use in further studies examining functional myelination and derivation of oligodendrocytes from genetically engineered ESC lines, including human ESCs. Results from the model presented here demonstrate a unique method for examining transplantable oligodendrocyte progenitors derived from ESCs for repair of white matter disease.
\end{abstract}

\section{KEY WORDS • central nervous system • cell differentiation • neural transplantation • pluripotent cell • neural regeneration • rat}

If as little as 10 to $15 \%$ of white matter is spared after spinal cord injury, it may be enough to support mobility.,15 Strategies to preserve injured white matter, prevent further loss to delayed degeneration, and reconstitute cellular loss through either endogenous regeneration or cellular transplantation targeted at this portion of the injured spinal cord may be enough for sufficient functional gain, sensory and/or motor. $3,8,14,15,17$ At this time, transplantation of young oligodendrocytes may prove to be a pragmatic approach to cellular reconstitution, especially after the demyelination has become chronic. ${ }^{13}$ Oligodendrocyte progenitors are at an early enough stage to multiply and yet at a late enough stage to remain restricted to their glial lineage in vivo. ${ }^{11,12}$ One important source of these cells is ESCs, which are expandable, maintaining stability under appropriate culture conditions, and genetically flexible. We have reported that after exposure to retinoic acid for 4 days, an additional culture scheme can be used to allow neural induced ESCs to enter an intermediate stage, known as oligospheres. ${ }^{12}$ This preparatory method can allow high-yield production of oligodendrocyte progenitors for in vitro or in vivo experiments.

Abbreviations used in this paper: CNS = central nervous system; $\mathrm{ESC}=$ embryonic stem cell; GFP = green fluorescent protein.
By transplanting ESC-derived oligodendrocyte progenitors early (postnatal Day 2), we generated chimeric white matter regions within the intact spinal cord, a novel model for study of single, adult oligodendrocytes in situ. We examined survival and incorporation of these mouse-derived ESC oligodendrocytes at two different time points: 1) following the period favored for glial development (gliogenesis), ${ }^{10}$ and 2) after the rat makes the transition into the adult stage. Tracking these cells with genetic expression of GFP, we found that embryonic stem oligodendrocytes could survive and demonstrate anatomically appropriate integration.

\section{MATERIALS AND METHODS}

Preparation of ESC, Oligosphere, and Transplantable Oligodendrocyte Progenitors

Embryonic B5 cells (strain 129SVJ, Dr. Nagy, University of Toronto, Canada) obtained in male mice were maintained in stable conditions as previously described. ${ }^{9}$ Briefly, continuous GFP expression was selected by addition of G418 (gentamicin; $500 \mathrm{mg} / \mathrm{ml}$ ) during undifferentiated passaging. ${ }^{20}$ We then induced ESCs through the $4-/ 4+$ retinoic acid protocol as previously described. ${ }^{1,12,16,20}$ Next, we dissociated the 8-day staged embryoid bodies and transferred them to flasks in SATO medium and basic fibroblast growth factor for 2 days 
to enhance the glial lineage derivation. ${ }^{12}$ Separating oligodendrocytes from astrocytes by using a method based on adherence principles, we could suspend a large portion of oligodendrocyte progenitor cells and transfer them into new flasks for an additional 4 days for further oligosphere derivation. ${ }^{12}$ Oligospheres were partially dissociated with trypsin and reconstituted in ESC induction medium. Transplantable cells were then maintained briefly on ice before transplantation. Cells for in vitro studies were frozen as embryoid bodies or plated on monolayers for 9 days to verify transplantable progenitor populations.

\section{Cell Transplantation and Animal Care}

A total of 45 rat pups (male and female) were divided into three groups that included control (eight), sham-operated (eight), or ESCinjected animals (29). On postnatal Day 2, designated rat pups were anesthetized by placing them on ice for approximately 5 to 8 minutes, after which they received 50,000 ESCs as single cells suspended in 5 $\mu l$ of growth medium, which were injected over 3 minutes at the sixth thoracic level, as previously described. ${ }^{20}$ As opposed to the prior description by Vadivelu, et al., in our study finely drawn glass pipettes were placed around the injection needle to establish a smaller injection apparatus and to induce a minimally traumatic injury during injection procedures.

The procedures we performed were in accordance with the Laboratory Animal Welfare Act, Guide for the Care and Use of Laboratory Animals, and Guidelines and Policies for Rodent Survival Surgery provided by the Animal Studies Committee of Washington University School of Medicine in St. Louis, Missouri. Thermoregulation was maintained for 1 hour during recovery from anesthesia by using a temperature-controlled chamber set at $36^{\circ} \mathrm{C}$. The rat pups were then returned to their mother and housed (12:12-hour light/dark cycle) until they were weaned at the adult stage and required separate cages.

\section{Tissue Fixation and Histological Investigation}

The rats were killed at either 3 or 9 weeks posttransplantation by using $4 \%$ paraformaldehyde/buffer, and then they were snap frozen in $-40^{\circ} \mathrm{C}$ isopentane and stored at $-70^{\circ} \mathrm{C}$ until sectioned. ${ }^{20}$ Tissue sections were cut into 14 - or $30-\mu \mathrm{m}$-thick sections and processed for immunofluorescence studies, using mouse marker labeling for verification.

\section{RESULTS}

\section{Transplantable ESC-Derived Oligodendrocyte Progenitors}

After 14 days of preparation, we examined in vivo the oligodendrocyte progenitors derived through oligosphere maturation steps (Fig. 1). Within the oligospheres we could identify a large yield of NG2-immunopositive cells (NG2 is a marker largely known for early-stage cells of the oligodendrocyte lineage). A second set of oligospheres was dissociated and plated on astrocyte monolayers for 1 day (the time recognized to allow these cells to stick to the monolayer). Here also, we found that a large proportion of cells was immunoreactive for the NG2 marker. The morphological features of these cells were consistent with bipolar, immature oligodendrocytes (unpublished observations).

\section{Participation in Neonatal Gliogenesis}

We transplanted approximately 50,000 oligosphere prepared ESCs into the intact spinal cord of rat pups during postnatal Day 2. We found that the neonatal spinal cord was suitable for direct injections, a procedure that does not require a prior laminectomy. After the injection, we returned the pups to their cages with their mother until weaning. In each repetition of our experiment series, a few rat pups (a total of five) were examined 3 days posttransplantation to further our goal of assessing the accuracy of the injection site with each repetition. Injection site locations were identified in $80 \%$ of these animals that were examined early.

At 3 weeks posttransplantation, we examined 12 postnatal Day 23 rat pups for histologically identified injury based on $\mathrm{H} \& \mathrm{E}$ staining patterns and apparent tissue morphological features. We found that eight of 12 animals had minimal injury at the sites (tissue damage that resembled a needle insertion site only), which were only found in gray matter and not white matter.

Tissue sections were also examined for single-cell survival of GFP-expressing oligodendrocytes. We found that greater than $90 \%$ of the surviving cells were located within white rather than gray matter, despite the variability in total number of cells surviving across individuals in the experimental group of animals. Surviving cells were widely distributed (located up to $5 \mathrm{~mm}$ apart from each other, up to $1.5 \mathrm{~cm}$ from the injection site). Single cells were aligned in a parallel orientation, and multiple extensions were aligned with presumed numerous parallel axons (Fig. 2). With appropriate anatomical integration, transplanted ESC oligodendrocyte progenitors appeared capable of participating in gliogenic events in situ.

\section{Maturation of Donor ESC-Derived Oligodendrocyte Progenitors Into Adult Oligodendrocytes}

We allowed a second group of animals to survive until 9 weeks posttransplantation. Our goal was to examine the adult rat spinal cord tissue for ESC-derived oligodendrocyte survival and anatomically appropriate integration of these donor oligodendrocytes. Using this early transplantation model, we found chimeric white matter into the adult stages. Although fewer total numbers of cells survived, all

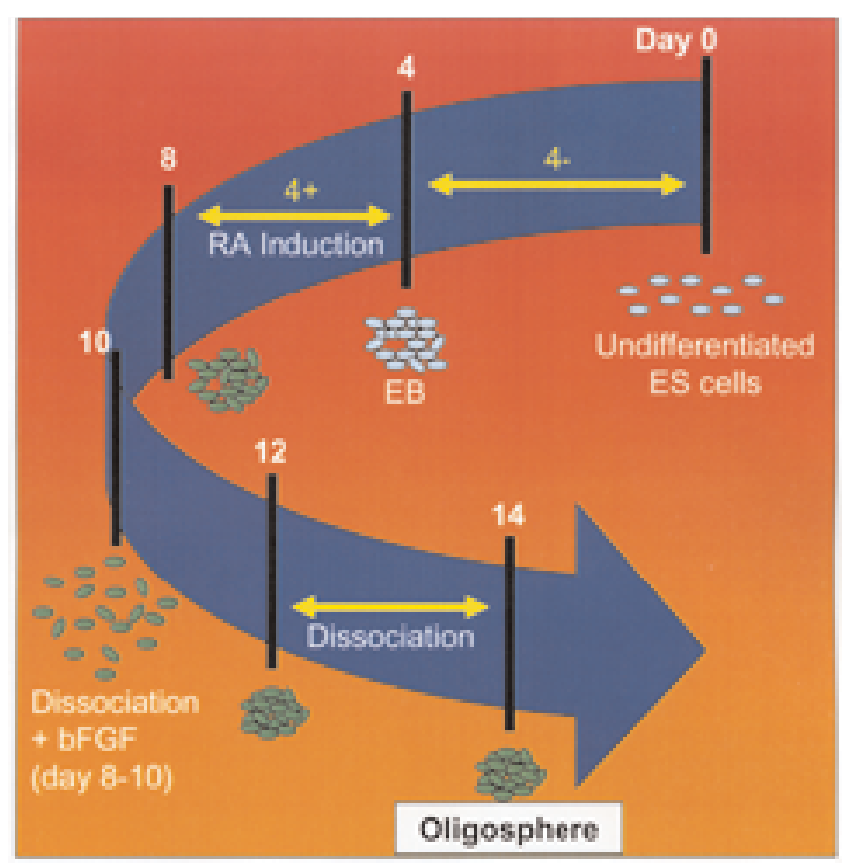

Fig. 1. Schematic illustration outlining the derivation of transplantable oligodendrocyte progenitors from ESCs (ES cells). $\mathrm{bFGF}=$ basic fibroblast growth factor; $\mathrm{EB}=$ embryoid body; $\mathrm{RA}=$ retinoic acid. 

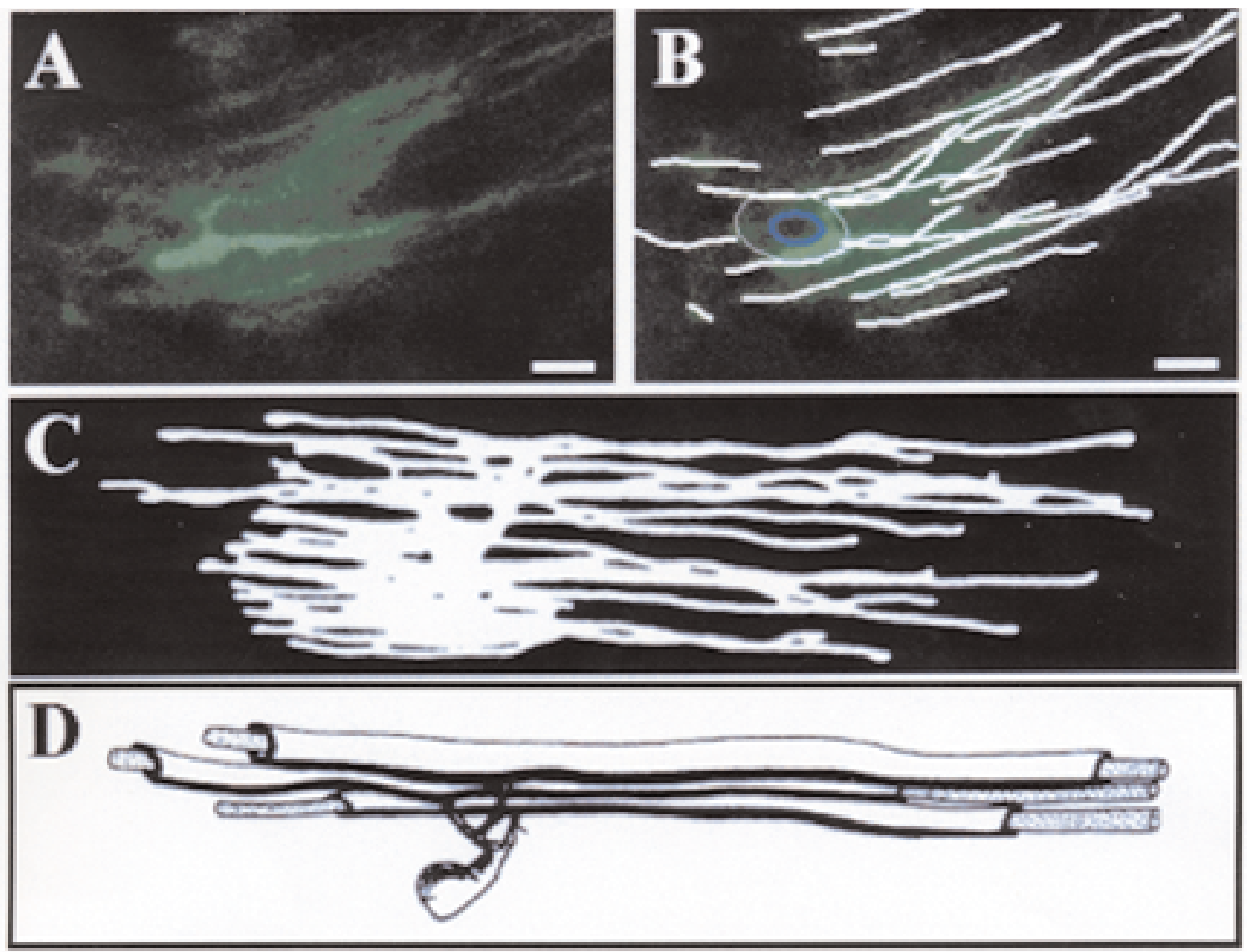

Fig. 2. Anatomical integration of ESC-derived oligodendrocytes. A: Photomicrograph showing GFP-expressing oligodendrocytes observed in white matter with multiple arms oriented in parallel alignment 3 weeks posttransplantation. B: Schematic illustration of the parallel segments of myelination based on the GFP expression shown in panel A. C and D: Schematic representations of CNS oligodendrocytes. These schematic drawings illustrate the normal pattern of myelination from a single oligodendrocyte in white matter. Note the resemblance in parallel arrangement of myelin segments. Scale bars $=10 \mu \mathrm{m}$.

of those identified were well distributed (not located in clusters) and appeared only within this adult rat white matter. Each GFP-expressing cell appeared as a mature oligodendrocyte with several extended arms that exhibited GFPexpressed rings at their ends, raising the possibility of axon wrapping (Fig. 3 and Video 1). Here, oligosphere ESCs were capable of surviving for a long time and maturing into adult oligodendrocytes in situ.

Video 1. Supplemental video file of the adult oligodendrocyte, three-dimensionally coded, for greater depth of view and with rotation shown through animation sequencing. (Click here to view with Windows Media Player, or here to view with RealPlayer.)

\section{DISCUSSION}

Our results demonstrate a unique method for examining transplantable oligodendrocyte progenitors derived from ESCs for their potential to repair white matter injury. We prepared ESCs through an additional selective maturation stage, the oligosphere, to yield a high percentage of transplantable oligodendrocyte progenitors. Prior genetic modification of these ESCs enabled us subsequently to identify ESC-derived mature, adult oligodendrocytes in situ, without the use of surface markers. Examination at 3 and 9 weeks posttransplantation demonstrated mature oligodendrocytes that integrated anatomically in an appropriate manner (participation through gliogenesis and development to adult maturity).

Chimeric models for the study of oligodendrocytes become important in addressing the question of identification. Today, all markers used to identify oligodendrocytes are not specific to the oligodendrocytes alone and label either the proteins produced or yield variable surface labeling, limiting the ability to identify and study these cells accurately. ${ }^{18}$ Our goal was to generate a targeted chimeric area to identify an adult oligodendrocyte accurately. Using an early injection model (postnatal Day 2), we were able to create targeted regions of chimeric spinal cord. Other investigators have reported extensive results after examining a similar approach with ESC transplantation through intrauterine injections for the purpose of following seeding patterns that generate widespread CNS chimerism. ${ }^{4,5}$ Our simple model enables us to focus on a targeted region of the spinal cord, generating only a local area of chimeric tissue. This was not associated with behavioral impairment as assessed with open field locomotor testing (Basso-BeattieBresnahan scale ratings, tracing experiments of explorato- 


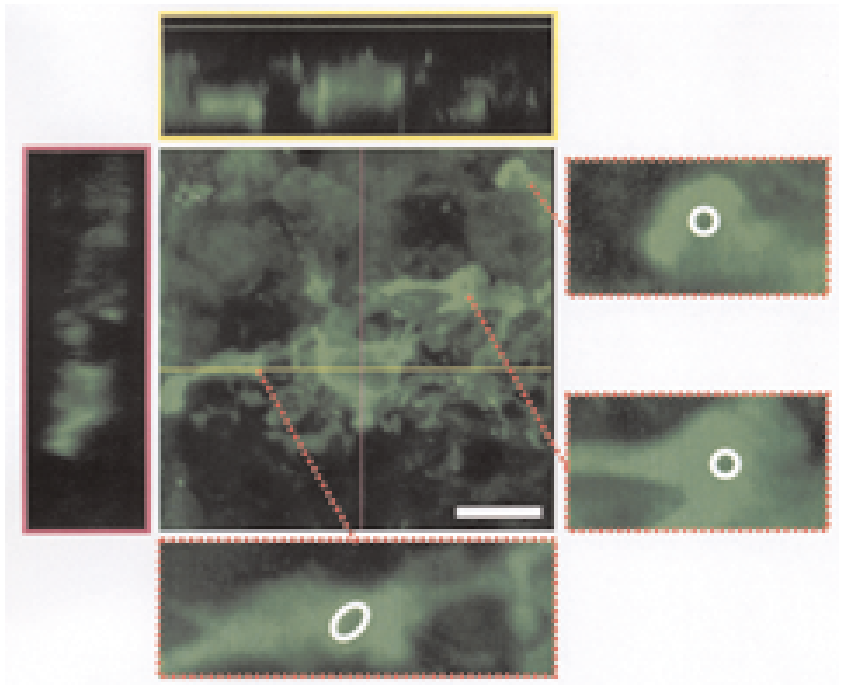

Fig. 3. Photomicrographs showing GFP-expressing adult oligodendrocyte in situ. Three-dimensional oligodendrocyte captured by confocal laser microscopy with respective orthogonal axis views. The red dotted boxes are magnified views on myelin ring profiles indicated by red dotted lines in the main image. Schematic overlays were used to highlight myelin ring profiles. Scale bar $=10 \mu \mathrm{m}$.

ry behavior, and rotarod training; our unpublished observations).

We were able to identify single oligodendrocytes by following their genetic expression of GFP. Unfortunately, unstable, long-term GFP expression may have given us an underestimated count of surviving oligodendrocytes. With this limitation in mind we are now able to test other hypotheses such as the use of genetic engineering approaches to promote oligodendrocyte survival, that is, transplantation of knockin/knockout donor cells $s^{7,21}$ or hosts. ${ }^{6,19}$ In addition, functional studies can now be used to examine the electrophysiological properties of single oligodendrocytes in situ that can be easily identified by GFP expression.

Using genetic expression of GFP, we were able to track oligodendrocytes into maturity. This result enabled us to identify a true adult oligodendrocyte with GFP tracking in situ and without assumed identification with cell body surface markers or markers against oligodendrocyte-produced proteins. In our study, this model enables future observations of the intrinsic developmental characteristics and interactions of oligodendrocytes with the environment (that is, cell-cell, cell-matrix), or immune signaling, in intact or injured spinal cord.

Experiments such as this one are greatly needed to understand the potential of stem cell transplantation in the treatment of CNS disease and to open doors to understanding the limited derivation of oligodendrocytes from human ESCs in vivo 22 and examinations of their role in functional myelination. In the near future, the transition of neural stem cell transplantation from basic science to clinical intervention may prove neurologically advantageous.

\section{CONCLUSIONS}

To our knowledge, this is the first time that an adult oligodendrocyte has been identified in situ, with three-dimen- sional imaging and without empirical, surface marker labeling. We transplanted an oligosphere, with staged ESCderived oligodendrocyte progenitors early into the neonatal spinal cord. At both the 3- and 9-week posttransplantation survival points, we could identify targeted regions of chimeric spinal cord. Using this principle opens doors for future studies identifying single oligodendrocytes, examining functional single cell-level receptor properties, and functional myelination. Our novel model presents a simple approach that can be used to begin examining the functional role of ESC-derived oligodendrocytes, including derivation and functional role from human ESCs, for application to white matter disease and repair.

\section{Acknowledgments}

We thank Drs. Shadi Farhangrazi, Michael J. Howard, and James Lu for critical discussions and Drs. Su Liu, Aiwu Lu, and Sathya Vadivelu for technical expertise.

\section{References}

1. Bain G, Gottlieb DI: Neural cells derived by in vitro differentiation of P19 and embryonic stem cells. Perspect Dev Neurobiol 5:175-178, 1998

2. Blight AR: Cellular morphology of chronic spinal cord injury in the cat: analysis of myelinated axons by line-sampling. Neuroscience 10:521-543, 1983

3. Blight AR: Miracles and molecules-progress in spinal cord repair. Nat Neurosci 5 (Suppl):1051-1054, 2002

4. Brustle O: Building brains: neural chimeras in the study of nervous system development and repair. Brain Pathol 9:527-545, 1999

5. Brustle O, Choudhary K, Karram K, et al: Chimeric brains generated by intraventricular transplantation of fetal human brain cells into embryonic rats. Nat Biotechnol 16:1040-1044, 1998

6. Dong H, Fazzaro A, Xiang C, et al: Enhanced oligodendrocyte survival after spinal cord injury in Bax-deficient mice and mice with delayed Wallerian degeneration. J Neurosci 23:8682-8691, 2003

7. Fedele DE, Koch P, Scheurer L, et al: Engineering embryonic stem cell derived glia for adenosine delivery. Neurosci Lett 370:160-165, 2004

8. Goldman S: Stem and progenitor cell-based therapy of the human central nervous system. Nat Biotechnol 23:862-871, 2005

9. Hadjantonakis AK, Gertsenstein M, Ikawa M, et al: Generating green fluorescent mice by germline transmission of green fluorescent ES cells. Mech Dev 76:79-90, 1998

10. Hirano M, Goldman JE: Gliogenesis in rat spinal cord: evidence for origin of astrocytes and oligodendrocytes from radial precursors. J Neurosci Res 21:155-167, 1988

11. Levison SW, Chuang C, Abramson BJ, et al: The migrational patterns and developmental fates of glial precursors in the rat subventricular zone are temporally regulated. Development 119:611-622, 1993

12. Liu S, Qu Y, Stewart TJ, et al: Embryonic stem cells differentiate into oligodendrocytes and myelinate in culture and after spinal cord transplantation. Proc Natl Acad Sci USA 97:6126-6131, 2000

13. Mason JL, Toews A, Hostettler JD, et al: Oligodendrocytes and progenitors become progressively depleted within chronically demyelinated lesions. Am J Pathol 164:1673-1682, 2004

14. McDonald JW: Repairing the damaged spinal cord. Sci Am 281:64-73, 1999

15. McDonald JW: Repairing the damaged spinal cord: from stem cells to activity-based restoration therapies. Clin Neurosurg 51:207-227, 2004

16. McDonald JW, Liu XZ, Qu Y, et al: Transplanted embryonic 


\section{Generating chimeric spinal cord}

stem cells survive, differentiate and promote recovery in injured rat spinal cord. Nat Med 5:1410-1412, 1999

17. Myckatyn TM, Mackinnon SE, McDonald JW: Stem cell transplantation and other novel techniques for promoting recovery from spinal cord injury. Transpl Immunol 12:343-358, 2004

18. Ness JK, Valentino M, McIver SR, et al: Identification of oligodendrocytes in experimental disease models. Glia 50:321-328, 2005

19. Rosenzweig ES, McDonald JW: Rodent models for treatment of spinal cord injury: research trends and progress toward useful repair. Curr Opin Neurol 17:121-131, 2004

20. Vadivelu S, Platik MM, Choi L, et al: Multi-germ layer lineage central nervous system repair: nerve and vascular cell generation by embryonic stem cells transplanted in the injured brain. J Neurosurg 103:124-135, 2005

21. Xian H, Gottlieb DI: Dividing Olig2-expressing progenitor cells derived from ES cells. Glia 47:88-101, 2004
22. Zhang SC, Wernig M, Duncan ID, et al: In vitro differentiation of transplantable neural precursors from human embryonic stem cells. Nat Biotechnol 19:1129-1133, 2001

Manuscript received August 1, 2005.

Accepted in final form August 18, 2005.

This work was supported by Grant Nos. NS39577 and NS36265 from the National Institute of Neurological Disorders and Stroke of the National Institutes of Health, and by a grant from the Christopher Reeve Paralysis Foundation to Dr. McDonald.

Address reprint requests to: John W. McDonald, M.D., Ph.D., The International Center for Spinal Cord Injury, Kennedy-Krieger Institute, 707 North Broadway, Baltimore, Maryland 21205. email: mcdonaldj@kennedykrieger.org. 